\title{
Evidence for He I $10830 \AA$ Absorption during the Transit of a Warm Neptune around the M-dwarf GJ 3470 with the Habitable-zone Planet Finder
}

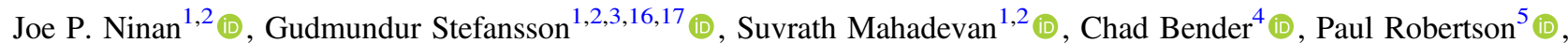 \\ Lawrence Ramsey $^{1,2}$, Ryan Terrien ${ }^{6}\left(\mathbb{0}\right.$, Jason Wright ${ }^{1,2,7}$ (1) , Scott A. Diddams ${ }^{8,9}$, Shubham Kanodia ${ }^{1,2}$ (1), William Cochran $^{10}$ (1), \\ Michael Endl ${ }^{10}$ (1) , Eric B. Ford ${ }^{1,2,11}$ (1), Connor Fredrick ${ }^{8,9}$, Samuel Halverson ${ }^{12}$ (1), Fred Hearty ${ }^{1,2}$, Jeff Jennings ${ }^{8,9}$, Kyle Kaplan ${ }^{4}$, \\ Emily Lubar ${ }^{10}$, Andrew J. Metcalf ${ }^{8,9,13}$, Andrew Monson ${ }^{1,2}$, Colin Nitroy ${ }^{1,2}$, Arpita Roy ${ }^{14,18}$ (i) , and Christian Schwab ${ }^{15}$ (1) \\ ${ }^{1}$ Department of Astronomy \& Astrophysics, 525 Davey Laboratory, The Pennsylvania State University, University Park, PA 16802, USA; jpn23@psu.edu \\ ${ }^{2}$ Center for Exoplanets and Habitable Worlds, 525 Davey Laboratory, The Pennsylvania State University,University Park, PA 16802, USA \\ Department of Astrophysical Sciences, Princeton University, 4 Ivy Lane, Princeton, NJ 08540, USA \\ ${ }^{4}$ Steward Observatory, University of Arizona, Tucson, AZ 85721, USA \\ ${ }^{5}$ Department of Physics and Astronomy, University of California-Irvine, Irvine, CA 92697, USA \\ ${ }^{6}$ Department of Physics and Astronomy, Carleton College, Northfield, MN 55057, USA \\ ${ }^{7}$ Penn State Astrobiology Research Center, University Park, PA 16802, USA \\ ${ }^{8}$ National Institute of Standards and Technology, 325 Broadway, Boulder CO 80305, USA \\ ${ }^{9}$ Department of Physics, University of Colorado, 2000 Colorado Avenue, Boulder CO 80309, USA \\ ${ }^{10}$ Department of Astronomy and McDonald Observatory, University of Texas at Austin, 2515 Speedway, Stop C1400, Austin, TX 78712, USA \\ ${ }^{11}$ Institute for CyberScience, The Pennsylvania State University, 525 Davey Laboratory, University Park, PA 16802, USA \\ 12 Jet Propulsion Laboratory, California Institute of Technology, 4800 Oak Grove Drive, Pasadena, CA 91109, USA \\ ${ }^{13}$ Space vehicles Directorate, Air Force Research Laboratory, 3550 Aberdeen Avenue SE, Kirtland AFB, NM 87117, USA \\ ${ }^{14}$ California Institute of Technology, 1200 E California Boulevard, Pasadena, CA 91125, USA \\ ${ }^{15}$ Department of Physics and Astronomy, Macquarie University, Balaclava Road, North Ryde, NSW 2109, Australia \\ Received 2019 October 4; revised 2020 March 26; accepted 2020 March 30; published 2020 May 11
}

\begin{abstract}
Understanding the dynamics and kinematics of outflowing atmospheres of hot and warm exoplanets is crucial to understanding the origins and evolutionary history of the exoplanets near the evaporation desert. Recently, groundbased measurements of the meta-stable helium atom's resonant absorption at $10830 \AA$ has become a powerful probe of the base environment which is driving the outflow of exoplanet atmospheres. We report evidence for the He I $10830 \AA$ in absorption (equivalent width $\sim 0.012 \pm 0.002 \AA$ ) in the exosphere of a warm Neptune orbiting the M-dwarf GJ 3470, during three transits using the Habitable Zone Planet Finder near-infrared spectrograph. This marks the first reported evidence for He I $10830 \AA$ atmospheric absorption for a planet orbiting an M-dwarf. Our detected absorption is broad and its blueshifted wing extends to $-36 \mathrm{~km} \mathrm{~s}^{-1}$, the largest reported in the literature to date. We modeled the state of helium atoms in the exosphere of GJ3470b based on assumptions on the UV and X-ray flux of GJ 3470, and found our measurement of flux-weighted column density of meta-stable state helium $\left(N_{\mathrm{He}_{3}^{2} \mathrm{~S}}=2.4 \times 10^{10} \mathrm{~cm}^{-2}\right)$, derived from our transit observations, to be consistent with the model, within its uncertainties. The methodology developed here will be useful to study and constrain the atmospheric outflow models of other exoplanets like GJ 3470b, which are near the edge of the evaporation desert.
\end{abstract}

Unified Astronomy Thesaurus concepts: Exoplanet atmospheric composition (2021); Exoplanet atmospheres (487); Exosphere (499); High resolution spectroscopy (2096); Near infrared astronomy (1093); Exoplanet astronomy (486)

\section{Introduction}

The conventional probe for escaping atmospheres has been the $\operatorname{Ly} \alpha$ absorption from the ionized exosphere during a planetary transit. This technique has produced exosphere discoveries around hot Jupiters, and hot and warm Neptunes, e.g., HD 209458b (Vidal-Madjar et al. 2003), GJ 436b (Kulow et al. 2014), and GJ 3470b (Bourrier et al. 2018). Ehrenreich et al. (2015) mapped an extended comet-like trail of escaping atmosphere from GJ 436b using the absorption signatures in the blue wings of Ly $\alpha$. Bourrier et al. (2018) performed a similar analysis for GJ 3470b using Ly $\alpha$ observations from the Hubble Space Telescope (HST) and detected an extended exosphere with neutral hydrogen around GJ 3470b.

\footnotetext{
16 NASA Earth and Space Science Fellow.

${ }^{17}$ Henry Norris Russell Fellow.

18 Millikan Prize Postdoctoral Fellow.
}

While Ly $\alpha$ is a powerful probe of evaporating atmospheres, it has two major drawbacks. The extinction loss due to interstellar absorption of $\mathrm{Ly} \alpha$, as well as the contamination from geocoronal emission, render the central core of the $\operatorname{Ly} \alpha$ line unusable. This implies one can probe only the high velocity regions of the exospheres via fitting the wings of the Ly $\alpha$ line. The UV observations also have to be done from space-above the Earth's atmosphere-rendering them expensive and hard to do for a large number of systems.

Oklopčić \& Hirata (2018) recently suggested the absorption lines of a metastable state of helium at $10830 \AA$ as an alternative probe of evaporating exoplanet atmospheres. He I $10830 \AA$ lines are not affected by the interstellar medium, and are observable from the ground using high-resolution nearinfrared spectrographs. Since the core of the line is accessible, even the low velocity base regions of the outflowing exosphere are detectable. High-resolution spectra enable us to isolate the stellar spectrum from the planet's absorption spectrum, which is modulated by the radial velocity of the planet. High 
resolution is also crucial for removing contamination from narrow telluric absorption lines for ground-based observations.

The search for He I $10830 \AA$ absorption is not new. Seager \& Sasselov (2000) proposed that this would be a large signature in an F9V type star, HD 209458, though a search by Moutou et al. (2003) with VLT/ISAAC did not yield a detection. Turner et al. (2016) had identified He I $10830 \AA$ along with other lines as a potential transition for transit spectroscopy. Recently, a handful of detections were made successfully around K-star planets, namely WASP-107b (Spake et al. 2018; Allart et al. 2019), HD 189733 b (Salz et al. 2018), HAT-P-11b (Allart et al. 2018; Mansfield et al. 2018), and WASP-69b (Nortmann et al. 2018), and one G-star planet HD 209458b (Alonso-Floriano et al. 2019).

In this paper, we report the evidence for He I $10830 \AA$ during the transit of GJ 3470b, a warm Neptune orbiting an M-dwarf star, using the Habitable-zone Planet Finder (HPF) on the $10 \mathrm{~m}$ Hobby-Eberly Telescope (HET) at the McDonald Observatory. Section 2 outlines our HPF observations. We discuss our He I $10830 \AA$ results and associated modeling in Section 3, and we finally summarize our key conclusions in Section 4.

\section{Observations and Data Reduction}

We observed GJ 3470 at different phases of GJ 3470b's orbit using the HPF spectrograph (Mahadevan et al. 2014; Metcalf et al. 2019). HPF is a near-infrared precision radial velocity spectrograph covering the wavelength regime of $8079-12786 \AA$ at a resolution of $R \sim 55,000$. HPF is actively temperature controlled to the $\mathrm{mK}$ level to enable exquisite spectral stability and precision radial velocities in the NIR (Stefansson et al. 2016). Due to the HET design (Ramsey et al. 1998; Shetrone et al. 2007), we are limited to observe for a track length of $\sim 1 \mathrm{hr}$ per night at GJ 3470's decl. We observed three transits of GJ 3470b on 2018 November 30 UT, 2019 January 19 UT, and 2019 April 16 UT, when the transits aligned with the observable window of the HET. Out-of-transit observations were conducted on 2019 January 4 UT, 2019 January 27 UT, and 2019 April 17 UT. Three frames of $916 \mathrm{~s}$ integration time were taken in each of the $\sim 1 \mathrm{hr}$ tracks during in-transit and out-transit observations. The median signal-tonoise ratio of individual spectra was $\sim 100$ per pixel for the first transit, and $\sim 200$ for the second and third transit. The median number of pixels per resolution element in HPF is 2.8 .

Echelle spectra of GJ 3470 from HPF were reduced using our HPF spectral extraction pipeline. The pipeline first generates 2D flux images (in units of electrons per second) from the up-the-ramp H2RG readout data (Ninan et al. 2018). A formal pixel-by-pixel variance image is also generated and propagated through the pipeline. The simultaneous star, sky, and calibration fiber spectra (as well as variance estimates) are then extracted from this 2D image (Kaplan et al. 2018).

To minimize scattered light contamination, no simultaneous calibration light spectra were used during the observations. The wavelength calibration of the extracted spectra was done using custom built frequency stabilized laser comb (LFC; Metcalf et al. 2019) measurements taken throughout the night, inversely weighted by the time difference between science and LFC images following the methodology in Metcalf et al. (2019) and Stefansson et al. (2020). All of our analyses and plots are in vacuum wavelengths. The spectra are then flat corrected and deblazed.
Sky emission lines are subtracted using the simultaneous sky spectrum. This step also subtracts out the smooth background scattered light due to the proximity of the sky fiber to the star fiber image on the detector. A 7 pixel median filter smoothing was applied to the sky fiber data in regions devoid of emission lines to reduce the noise in continuum regions of the sky emission spectrum. Stellar continuum was also removed by fitting a quadratic polynomial continuum. Telluric absorption lines were corrected using an improved version of the TERRASPEC code (Bender et al. 2012), a wrapper around LBLRTM (Clough et al. 2005). A good sky emission line subtraction and telluric absorption correction was crucial since He I $10830 \AA$ falls close to strong OH emission lines as well as water absorption lines. The residuals in the sky and telluric corrected regions are still dominated by imperfect modeling and they are significantly above the photon noise.

Due to HET's constrained pointing-resulting in short $(\sim 1$ hr long) observation windows on GJ 3470 — our out-of-transit and in-transit observations are spread across multiple nights. To down-weight the region of the spectrum partially recovered from sky and telluric corrections, the variances of those regions are artificially inflated by a large factor $(\sim 100)$. In doing so, this allows us to combine multiple epochs (with different barycentric shifts) weighted by the variance and obtain an average spectrum without residual artifacts from imperfect telluric and sky emission subtraction dominating.

The GJ 3470 system's parameters used in this paper are summarized in Table 1.

\section{Results and Discussion}

\subsection{Evidence for He I $10830 \AA$ A Absorption during Transits}

The blue curve at the top of Figure 1 shows the weighted average of all the in-transit spectra from three transit epochs divided by the average of the out-of-transit spectra. The vacuum wavelengths of the He $10830 \AA$ triplet in the planet's rest frame are marked by the orange vertical lines. Since the telluric correction as well as the sky emission line subtraction are not perfect, regions of sky/telluric contamination have enlarged error bars as discussed in Section 2. The weighted average of all combinations of transits taken two at a time is also shown in the curves below. The single peak observed at $10833 \AA$ is not present in Transits 2 and 3 , it is only seen in Transit 1. However, since $10833 \AA$ was separated from the telluric band only during Transit 1 , it did not get averaged out in the final weighted average. Since Transit 1 had only half the $\mathrm{S} / \mathrm{N}$ compared to the other two transits we suspect this is an artifact due to photon and detector noise (see blue curves in Figure 5 for the individual ratio spectrum from Transit 1). For a null result comparison, one of the out-of-transit epochs is also shown in Figure 1. These data are processed the same way as other in-transit spectra, and we do not see any signatures of absorption inside the He $10830 \AA$ triplet window.

\subsection{Column Density Measurement from Equivalent Width (EW) Analysis}

We measure the integrated EW of the detected broad absorption in the vacuum wavelength range (10831.9 $\AA$ to $10833.6 \AA$ ) in Figure 1 to be $0.012 \pm 0.002 \AA$. We ignored the $J=0$ line (10832.06 $\AA$ in vacuum) of the triplet since its oscillator strength is only one-eighth of the other two lines combined, so it is below our continuum noise (the $J=0$ line is 
Table 1

Orbital Parameters of GJ 3470b Used in the Calculation

\begin{tabular}{lcll}
\hline \hline Parameter & Value & Description & References \\
\hline$\gamma$ & 26.090 & Absolute stellar RV $\left(\mathrm{km} \mathrm{s}^{-1}\right)$ & Gaia Collaboration et al. (2018) \\
$T_{\text {eff }}$ & 3600 & Stellar effective temperature (K) & Awiphan et al. (2016) \\
{$[\mathrm{Fe} / \mathrm{H}]$} & 0.20 & Stellar metallicity & Awiphan et al. (2016) \\
$\log g$ & 4.695 & Stellar surface gravity & Awiphan et al. (2016) \\
$M_{*}$ & $0.51 \pm 0.06$ & Stellar mass $\left(M_{\odot}\right)$ & Biddle et al. (2014) \\
$R_{*}$ & $0.48 \pm 0.04$ & Stellar radius $\left(R_{\odot}\right)$ & Biddle et al. (2014) \\
$T_{0}$ & $2456677.727712 \pm 0.00022$ & Transit midpoint (BJD & Dragomir et al. (2015) \\
$T_{14}$ & $0.07992 \pm 0.001$ & Transit duration (days) & Dragomir et al. (2015) \\
$P$ & $3.3366413 \pm 0.0000060$ & Period (days) & Dragomir et al. (2015) \\
$e$ & $0.114 \pm 0.052$ & Eccentricity & Kosiarek et al. (2019) \\
$\omega$ & $-1.44_{-0.04}^{+0.1}$ & Argument of periastron $(\mathrm{rad})$ & Kosiarek et al. (2019) \\
$K$ & $8.21_{-0.46}^{+0.47}$ & RV semi amplitude $\left(\mathrm{m} \mathrm{s}^{-1}\right)$ & Kosiarek et al. (2019) \\
$M_{\mathrm{pl}}$ & $12.58_{-1.28}^{+1.31}$ & Mass of the planet $\left(M_{\oplus}\right)$ & Kosiarek et al. (2019) \\
$b$ & $0.47_{-0.110}^{+0.074}$ & Impact Parameter & Dragomir et al. (2015) \\
\hline
\end{tabular}

the dashed orange line in Figure 1). The resonant scattering absorption of He I $10830 \AA$ is directly proportional to the number density of $\mathrm{He} \mathrm{I}$ in the ${ }_{3 \mathrm{~S}}^{2}$ metastable triplet state. We use the curve of growth analysis in the optically thin regime to estimate the column density of $\mathrm{He}_{3}^{2} \mathrm{~S}$ metastable atoms,

$$
N_{\mathrm{He}_{3}^{2} \mathrm{~S}}=\frac{\mathrm{EW}}{8.85 * 10^{-13}} \frac{1}{\lambda_{2}^{2} \times f_{i k_{2}}+\lambda_{3}^{2} \times f_{i k_{3}}} \mathrm{~cm}^{-2},
$$

where $N_{\mathrm{He}}{ }_{3}^{2} \mathrm{~S}$ is the column density of $\mathrm{He}{ }_{3}^{2} \mathrm{~S}$ metastable atoms in $\mathrm{cm}^{-2}$, EW is the measured combined EW of $J=1$ and $J=2$ lines of He $10830 \AA$ triplet in units of $\AA, \lambda_{2}$ and $\lambda_{3}$ are the wavelengths of the $J=1$ and $J=2$ triplet lines in units of microns, and $f_{i k_{2}}$ and $f_{i k_{3}}$ are the oscillator strengths of the lines taken from the NIST Atomic Spectra Database Lines Database (Drake 2006).

Substituting our measured EW, we obtain $N_{\mathrm{He}}{ }_{3}^{2} \mathrm{~S}=2.4 \times 10^{10} \mathrm{~cm}^{-2}$. This measured $\mathrm{EW}$ is a fluxweighted average across the unresolved stellar disk during the transit. Hence, the column density we measure is also a stellar disk flux-weighted average column density of the exosphere during the transit.

\subsection{Theoretical Column Density from an Exosphere Model}

We modeled the steady-state helium distribution in the outflowing exosphere of GJ 3470b using the formalism and state transition coefficients outlined in Oklopčić \& Hirata (2018). Using hydrodynamic simulations Salz et al. (2016) show that the exosphere of GJ $3470 \mathrm{~b}$ is not isothermal. We therefore do not use a Parker wind model for our atmospheric analysis, but solve for the steady-state helium distribution using the velocity and density field of the exosphere from the Salz et al. (2016) PLUTO-CLOUDY hydrodynamic simulations. To be internally consistent, we also used the GJ 3470 stellar SED used by Salz et al. (2016).

The X-ray spectrum of GJ 3470 we used from Salz et al. (2016) is calculated using the plasma emission model from CHIANTI (Dere et al. 1997, 2009) normalized to the X-ray luminosity of GJ $3470 \mathrm{~b}$ estimated based on the stellar rotation period and the stellar mass (Pizzolato et al. 2003). The EUV flux ( $100 \AA$ to $\sim 912 \AA$ ), which is critical for these calculations due to the strong dependence of helium ionization, comes from the scaling of the model-dependent Ly $\alpha$ flux (Linsky et al.
2014). The model-dependent Ly $\alpha$ flux of GJ 3470 itself is estimated by the Linsky et al. (2013) model based on the X-ray luminosity. See Section 2.2 of Salz et al. (2016) for more details. This model-dependent irradiance spectrum of GJ 3470 is the source of the biggest uncertainty in our calculations. However, the model-dependent Ly $\alpha$ flux Salz et al. (2016) derive, and the value we adopt here, is consistent with the Ly $\alpha$ flux measured by Bourrier et al. (2018) during three transits of GJ 3470b using the Space Telescope Imaging Spectrograph instrument on board the HST.

As most of the free electrons in the atmospheres of exoplanets come from ionized hydrogen, to obtain the electron density of the GJ 3470 atmosphere, we first solved for the steady-state of hydrogen following Oklopčić \& Hirata (2018). To obtain the steady-state distribution of helium atoms, we briefly discuss here the relevant system of integro-differential equations from Oklopčić \& Hirata (2018), given by,

$$
\begin{aligned}
v \frac{\partial f_{1}}{\partial r}= & \left(1-f_{1}-f_{3}\right) n_{e} \alpha_{1}+f_{3} A_{31}-f_{1} \Phi_{1} e^{-\tau_{1}} \\
& -f_{1} n_{e} q_{13 a}+f_{3} n_{e} q_{31 a}+f_{3} n_{e} q_{31 b}+f_{3} n_{\mathrm{H}^{0}} Q_{31},
\end{aligned}
$$

and,

$$
\begin{aligned}
v \frac{\partial f_{3}}{\partial r}= & \left(1-f_{1}-f_{3}\right) n_{e} \alpha_{3}-f_{3} A_{31}-f_{3} \Phi_{3} e^{-\tau_{3}} \\
& +f_{1} n_{e} q_{13 a}-f_{3} n_{e} q_{31 a}-f_{3} n_{e} q_{31 b}-f_{3} n_{\mathrm{H}^{o}} Q_{31},
\end{aligned}
$$

where $f_{1}$ and $f_{3}$ are the fractions of neutral helium in the ground state of the singlet and triplet states, respectively, $v$ is the velocity of the outflowing exosphere as a function of radius we adopt from Salz et al. (2016), $n_{e}$ is the density of free electrons we obtained by solving the steady-state of hydrogen, and $\alpha_{1}$ and $\alpha_{3}$ are the helium recombination rates to singlet and triplet state from Osterbrock \& Ferland (2006), respectively. The collision coefficients $q_{i j k}$ and $Q_{31}$ are from Oklopčić \& Hirata (2018), calculated using coefficients from Bray et al. (2000) and Roberge \& Dalgarno (1982). The radiative decay rate $A_{31}$ from the helium metastable state to singlet state is adopted from Drake (1971). $\Phi_{1}$ and $\Phi_{3}$ are the effective photoionization rate 


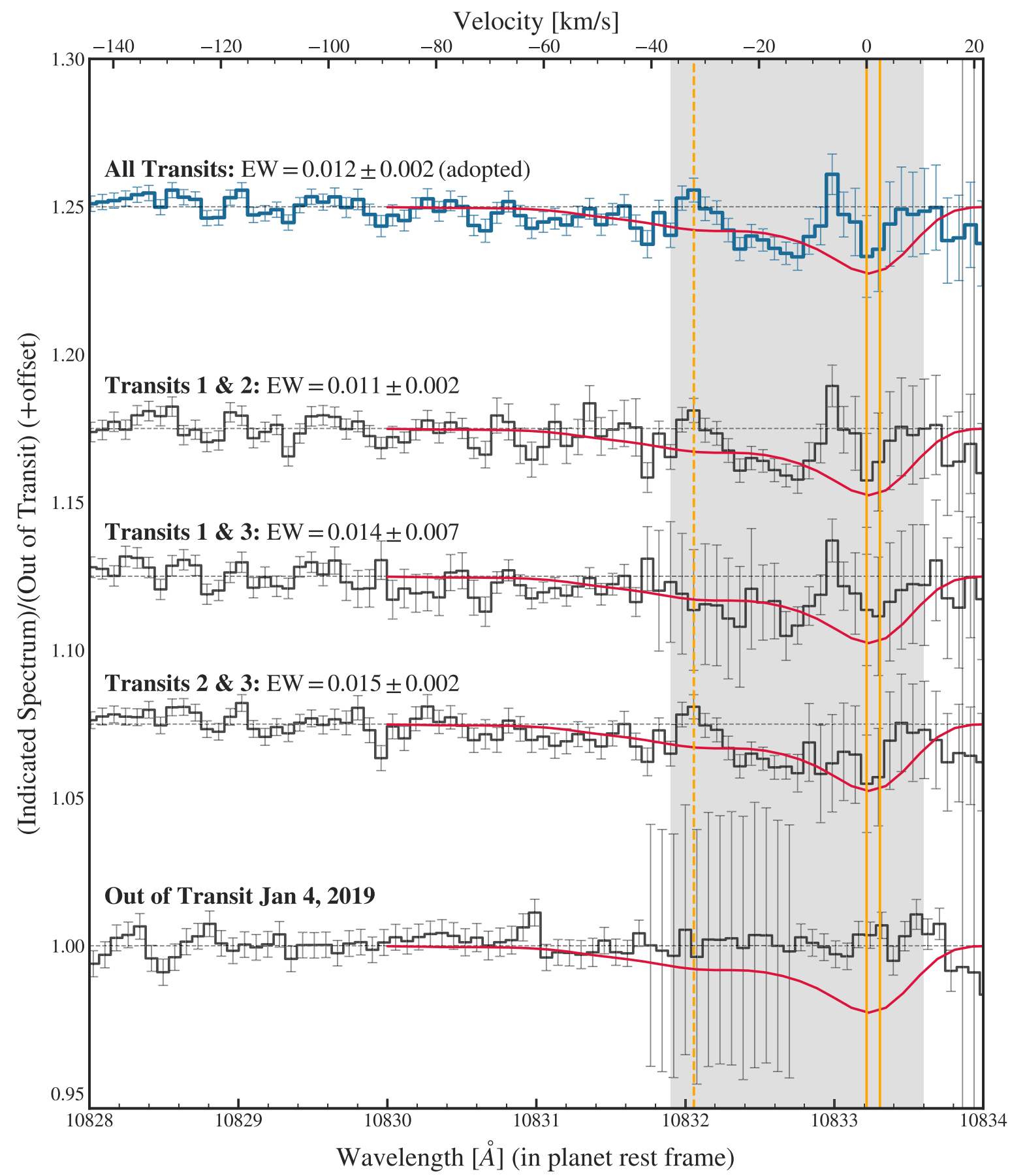

Figure 1. Evidence of broad He 10830 absorption (EW: $0.012 \pm 0.002 \AA$ ) in the wavelength range $10832.2-10833.4 \AA$ in the in-transit/out-transit spectrum during three transits of GJ 3470b. The $x$-axis shows vacuum wavelength in the planet's rest frame at midtransit. The rest vacuum wavelengths of the He $10830 \AA$ triplet lines in planet's rest frame are marked by the vertical dashed and solid orange lines. The gray region highlights the broad absorption discussed in this paper, and it is the wavelength window we used to measure reported equivalent widths. The expected line profile from our simple model described in Section 3.3 .1 is also shown for comparison by the red curves. An out-of-transit epoch divided by the reference out-of-transit spectrum is also shown at the bottom, demonstrating the lack of any absorption in out-of-transit data.

coefficients calculated using,

$$
\Phi_{i}=\int_{\lambda_{1}}^{\lambda_{2}} \frac{\lambda F_{\lambda}}{h c} a_{\lambda} d \lambda
$$

where $F_{\lambda}$ is the irradiated flux on GJ3470b described earlier in this section, $a_{\lambda}$ is the photoionization cross section taken from Brown (1971) for the singlet state, and from Norcross (1971) for the triplet state. For the singlet state, the integral is evaluated up to the ionization wavelength of helium (504 $\AA$ ), while for the triplet state the integral is computed in the interval starting at the Lyman $\operatorname{limit}^{19}(911.6 \AA)$ to the metastable state ionization wavelength $(2583 \AA)$.

\footnotetext{
${ }^{19}$ Photons of energy higher than the Lyman limit are many orders of magnitude more likely to be absorbed by the hydrogen than $\mathrm{He}_{3}^{2} \mathrm{~S}$, hence the beginning of the integral window was chosen to be at $911.6 \AA$.
} 

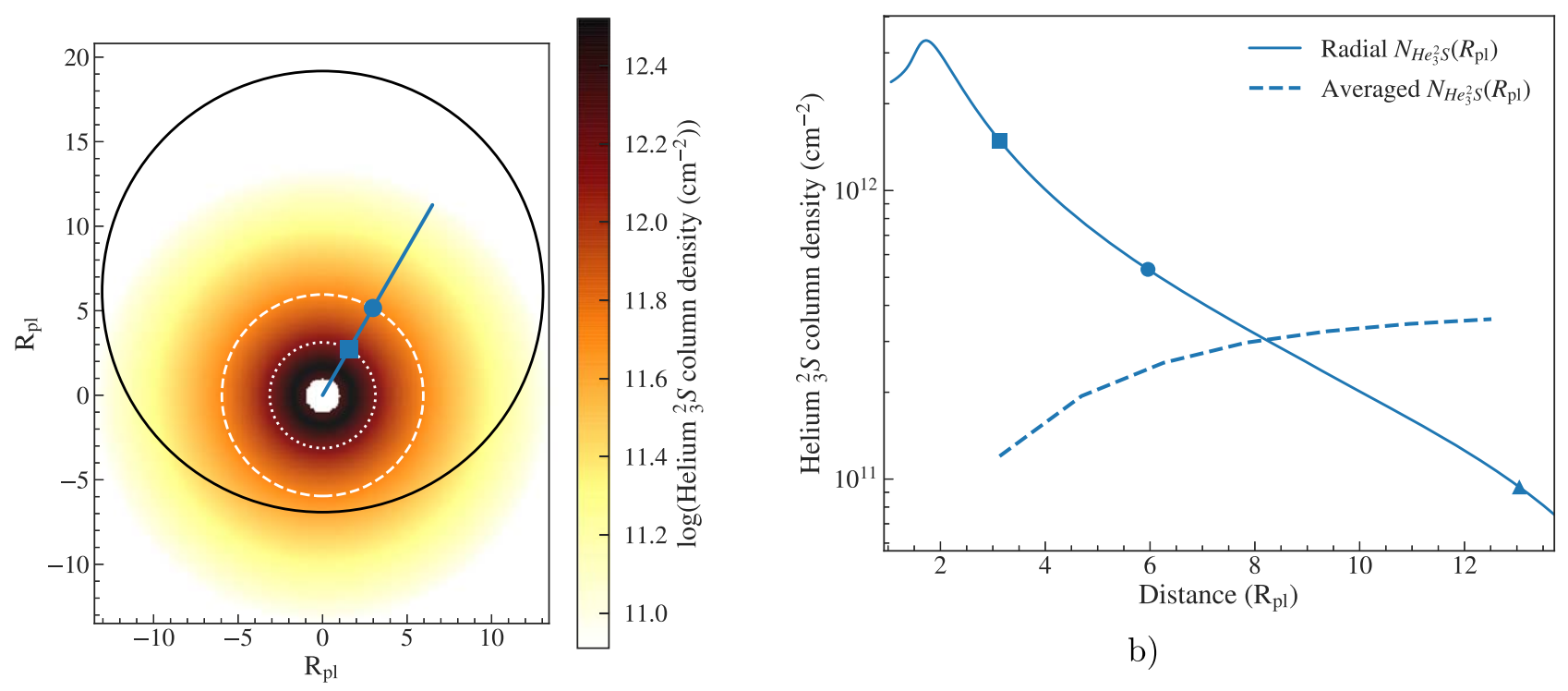

b)

a)

Figure 2. (a) The $2 \mathrm{D}$ diagram showing the projection of the Roche lobe radii as well as the GJ3470 stellar disk on top of the spherical 1D He ${ }_{3}^{2} \mathrm{~S}$ metastable atom column density map predicted by our 1D simulation of GJ 3470b. The volume equivalent Roche lobe radius as well as the Roche lobe extent on the star-planet axis is marked by the dotted and dashed circles respectively. The large circle marks the disk of the star. (b) The radial cut plot of the column density along the blue line in left figure is shown here as "Radial $N_{\mathrm{He}}{ }_{3} \mathrm{~s}$." The volume equivalent Roche lobe radius as well as the Roche lobe extend on the star-planet axis is marked by the square and dot respectively. The triangle marks the radius of the star. The dashed line shows the background stellar disk flux-averaged column density predicted by our model at the center of the transit. It is a function of the assumed extent of the exosphere beyond the volume equivalent Roche lobe as shown on left.

To calculate the optical depths $\tau_{1}$ and $\tau_{3}$ as a function of radius for the singlet and metastable states of helium, we compute the following integrals,

$$
\begin{aligned}
\tau_{1}= & a_{\mathrm{oH}} \frac{0.9}{1.297 * m_{p}} \int_{r}^{\infty}\left(1-f_{\mathrm{H}+}\right) \rho(r) d r \\
& +a_{\mathrm{oHe}} \frac{0.1}{1.297 * m_{p}} \int_{r}^{\infty} f_{1} \rho(r) d r,
\end{aligned}
$$

and,

$$
\tau_{3}=a_{\mathrm{oHe} 2 \mathrm{~S} 3} \frac{0.1}{1.297 * m_{p}} \int_{r}^{\infty} f_{3} \rho(r) d r
$$

where $m_{p}$ is the proton mass, $\rho(r)$ is the density of the GJ3470b exosphere from Salz et al. (2016), $f_{\mathrm{H}+}$ is the fraction of ionized hydrogen we obtained by solving the steady-state of hydrogen. $a_{\mathrm{oH}}$ and $a_{\mathrm{oHe}}$ are GJ3470b's irradiation flux-averaged hydrogen and helium ionization cross sections in the wavelength range up to helium ionization $(504 \AA) . a_{\mathrm{oHe} 2 \mathrm{~S} 3}$ is the flux-averaged cross section of $\mathrm{He}_{3}^{2} \mathrm{~S}$ in the wavelength range $911.6 \AA$ (hydrogen ionization) to $2583 \AA$ ( $\mathrm{He}_{3}^{2} \mathrm{~S}$ ionization).

We solve Equations (2) and (3) iteratively as an initial boundary value partial differential equation by starting with an initial estimate for the optical depth integral term (Equations (5) and (6)). Using the resulting solution, we updated the integral term in Equations (5) and (6). The final solutions converged within one or two iterations since the integral term has impact only in the high opacity base region of the outflowing atmosphere. The differential equation of $\mathrm{He}_{3}^{2} \mathrm{~S}$ metastable atoms (Equation (3)) is very stiff for high densities of GJ $3470 \mathrm{~b}$, and we therefore used a Radau solver with adaptive dense gridding (Hairer \& Wanner 1996).

The one-dimensional radial density distribution of $\mathrm{He}_{3}^{2} \mathrm{~S}$ metastable atoms obtained above is then integrated assuming a spherical exosphere to obtain column densities at different impact parameters from the planet (Figure 2(b)). We expect the model to fail beyond the Roche-lobe due to complex stellar wind interactions. We estimate the volume equivalent Rochelobe radius of the GJ $3470 \mathrm{~b}$ system to be $3.12 R_{p}$ following Eggleton (1983). The teardrop shaped Roche-lobe's extent on the star-planet axis is $5.96 R_{p}$ (Salz et al. 2016). Both of these points are explicitly highlighted in Figure 2. Assuming an impact parameter of $b=0.47$ (Dragomir et al. 2015), Figure 2(a) shows the 2D projection of the system during the midpoint of the transit. In Figure 2(a) we also illustrate the stellar disk on top of the column density map of $\mathrm{He}_{3}^{2} \mathrm{~S}$ metastable atoms. To estimate the flux-averaged column density, we used a quadratic limb-darkening model where we calculated the limb-darkening coefficients using the EXOFAST web-applet ${ }^{20}$ using the stellar effective temperature, metallicity, and surface gravity from Table 1 , in the $J$ band. This fluxweighted average column density is dependent on how far the exosphere extends beyond the Roche-lobe; the predicted background stellar disk flux-weighted average column density of $\mathrm{He}{ }_{3}^{2} \mathrm{~S}$ metastable atoms as a function of the extent of GJ 3470 b's exosphere is shown by the dashed line in Figure 2(b)).

\subsubsection{Line Profile Model}

The broad absorption we present evidence for during the transit of GJ $3470 \mathrm{~b}$ is possibly the broadest He $10830 \AA$ absorption reported in the literature to date. It spans a velocity range of -36 to $+9 \mathrm{~km} \mathrm{~s}^{-1}$. Figure 3(a) shows the outflowing exosphere velocity from Salz et al. (2016). The wind reaches only velocities up to $\sim 10 \mathrm{~km} \mathrm{~s}^{-1}$ inside $3.12 R_{p}$-the volume equivalent Roche-lobe radius. Both the volume equivalent

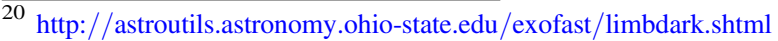



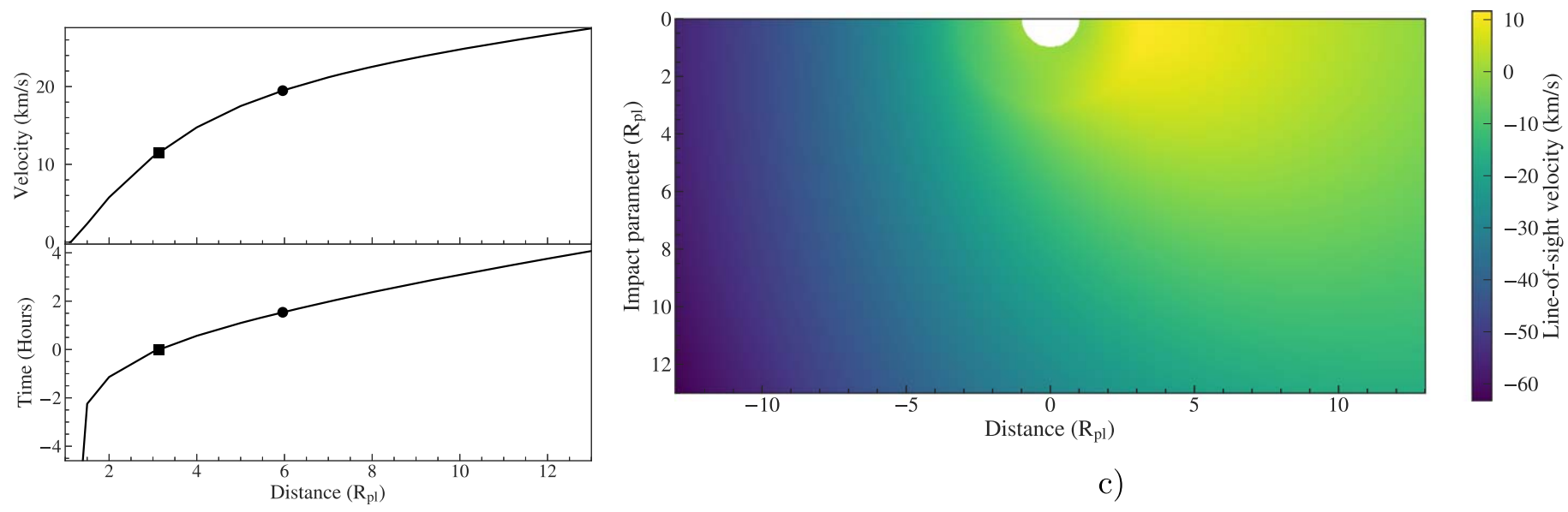

a), b)

c)

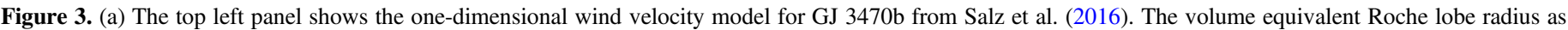

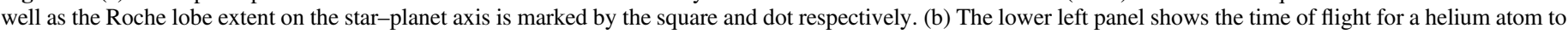

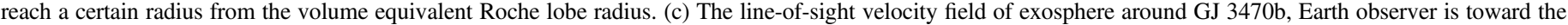
left side of the axis and the host star is toward the right side.

Roche lobe radius as well as the Roche lobe extent on the starplanet axis $\left(5.96 R_{p}\right)$ is marked by vertical lines in the plot.

The line-of-sight velocity of the helium atoms we see during the transit is the sum of the hydrodynamic driven wind velocity plus the stellar radiative acceleration, and the planet's orbital radial velocity at the instant of their escape from the planet's gravitational potential. For example, helium atoms escaping the planet potential $\sim 2.5 \mathrm{hr}$ before the transit will have a line-ofsight velocity of $\sim-30 \mathrm{~km} \mathrm{~s}^{-1}$ during the transit due to change in the line-of-sight component of planet's orbital velocity. Figure 3(b) shows the time a helium atom will take to travel the distance based on the wind model after it leaves the Roche lobe. Atoms that escape the potential $2.5 \mathrm{hr}$ before the transit will be at $\sim 8 R_{p}$, which is well within the projected stellar disk.

For calculating the line profile predicted by our model, we need to calculate the line-of-sight velocity field of the GJ $3470 \mathrm{~b}$ exosphere at all impact parameter positions and radial distances. For the region inside the volume equivalent Roche Lobe, we consider only the line-of-sight projection of the radial hydrodynamic wind velocity. For the region outside the Roche lobe, we added the line-of-sight component of the hydrodynamic wind to the difference in the planet's radial velocity between present and the time at which gas at a certain altitude escaped the Roche lobe. Figure 3(c) shows this velocity field. The observer on Earth is toward the left side of the axis and the host star is toward the right side. The negative line-of-sight velocity implies gas is moving toward the observer. In reality, there will be additional blueshift due to the interaction with stellar wind. We do not include any stellar wind induced blueshift of the exosphere in our line model.

To model the line profile, we used the Voigt profile. The modeling methodology is mostly similar to Oklopčić \& Hirata (2018), the only difference is in not assuming a spherically symmetric velocity field, and using the GJ 3470b system's impact parameter and stellar disk limb-darkening model while integrating the absorption line profile across the stellar disk. The temperature of the gas for the thermal Gaussian component of the modeled Voigt profile was taken to be $7000 \mathrm{~K}$ (Salz et al. 2016; Bourrier et al. 2018). The He 10830 line's Einstein coefficient for the Lorentzian component of the Voigt profile was taken from NIST Atomic Spectra Database Lines Database
(Drake 2006). The oscillator strengths for the cross section calculation of each triplet line is also taken from the NIST Database. These cross sections, along with the radial density distribution of the metastable helium we obtained in our model (Section 3.3) were integrated to obtain the optical depth at different impact parameter distances from the planet. Density of metastable helium outside $13 R_{\mathrm{pl}}$ (radial limit of our 1D model) was set to zero in our integrals. Using a quadratic limbdarkening model, we averaged the transmission curve across the disk to obtain the net transmission profile of the He 10830 triplet lines in GJ 3470b during the transit. Since the measured column density is less than the model predicted column density by a factor of 10 (see Section 3.4), we scaled down the density distribution from our model also by a factor of 10 . This predicted line profile is overplotted over all the transit and out of transit ratio spectra in Figure 1.

\subsubsection{Limits of the Model}

The simplified one-dimensional model we presented here is to check whether the strength of our claimed helium $10830 \AA$ absorption in the exoplanet atmosphere is within the limits of expected physical conditions. A true three-dimensional wind model where the stellar irradence is only on one side of the planet is known to have lesser density on average in all angles by a factor of 4 than the one-dimensional symmetric wind model we used (Stone \& Proga 2009).

The other major source of uncertainty in our model is the irradiated flux in the wavelengths shorter than helium ionization $(\lambda<512 \AA)$. As briefly outlined in the section above, it is estimated by a chain of empirical models. We expect the helium ionization flux to have a significant influence on the population of metastable He atoms. To study the impact, we simulated how the predicted column density of $\mathrm{He}_{3}^{2} \mathrm{~S}$ metastable atoms changes when we suppress the irradience at wavelength shorter than $504 \AA$ by different factors. ${ }^{21}$ Results

\footnotetext{
21 The reason we chose $504 \AA$ for this test instead of $512 \AA$ is because photons of energy higher than $504 \AA$ are more likely to ionize a helium atom than a hydrogen atom due to the factor of 10 lower abundance of helium than hydrogen. We want to probe the impact on helium ionization alone without significant change to hydrogen ionization.
} 


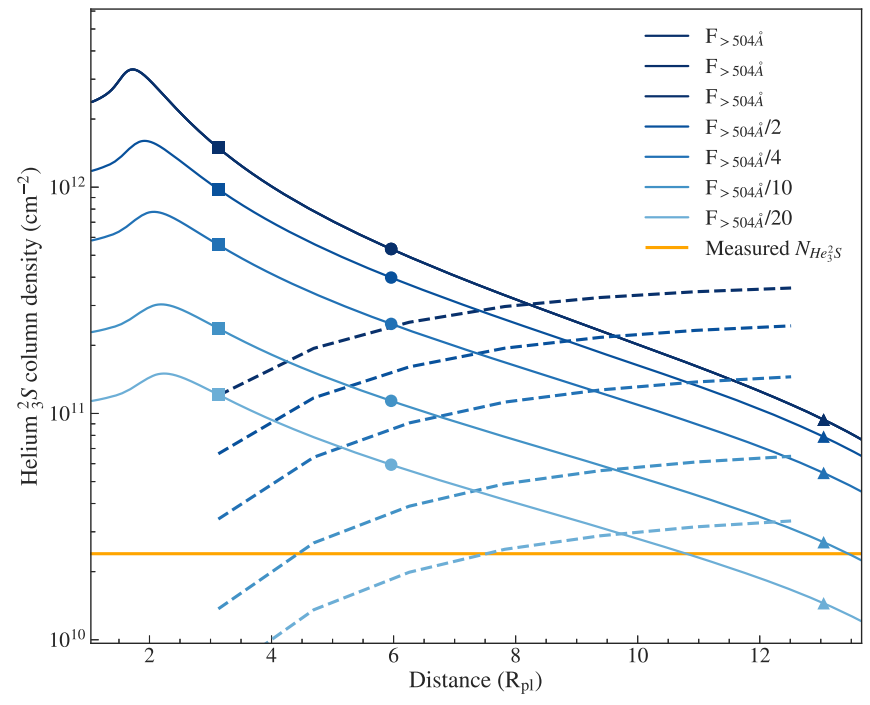

Figure 4. The column density of $\mathrm{He}_{3}^{2} \mathrm{~S}$ metastable atoms predicted by the $1 \mathrm{D}$ simulation of GJ $3470 \mathrm{~b}$ is reduced proportionally to the flux in wavelengths less than $504 \AA$ when everything else-including the density and velocity-is kept fixed. The volume equivalent Roche lobe radius as well as the Roche lobe extent on the star-planet axis is marked by the square and dot respectively. The triangle marks the radius of the star. The dashed lines show the flux-averaged column density predicted by this model at the center of the transit as a function of the extent of the exosphere beyond the volume equivalent Roche lobe. The horizontal orange line shows the measured average column density in transits of GJ 3470b using HPF.

are shown in Figure 4. Since we wanted to probe only the impact on the steady state of helium atoms, the underlying velocity field and density used in the differential equation was kept fixed, and is the same as the Salz et al. (2016) hydrodynamic model for the original irradiance. It is very instructive to see that the observed column density of metastable helium is reduced proportionally to the reduction in the helium ionization flux.

In real systems, since the mass outflow is proportional to the energy absorbed in the lower atmosphere from irradiance, there will be an additional proportional reduction in density when the EUV flux irradiation is reduced. Hence, the combined effect of reduction in helium ionizing radiation is quadratic on the column density of meta-stable helium atoms.

A few more ways the underlying density of the wind we used in our simulation from Salz et al. (2016) can be impacted is summarized in Table 2 of Salz et al. (2016).

\subsection{On the Difference between the Predicted and Measured Column Densities}

As described in Section 3.2, the measured flux-weighted column density of $\mathrm{He}_{3}^{2} \mathrm{~S}$ is $N_{\mathrm{He}}{ }_{3}^{2} \mathrm{~S}=2.4 \times 10^{10} \mathrm{~cm}^{-2}$. This is a factor of 10 less than predicted by our one-dimensional hydrodynamic model described in Section 3.3. However, as discussed in Section 3.3.2, if we correct our simulation for the 3D model simplification to the 1D model by the factor of 4 (Stone \& Proga 2009), and assume the helium ionization radiation is lesser by a factor of 1.6 (which is well within the error of the empirically derived EUV SED of GJ3470 in Salz et al. 2016), we reduce the predicted metastable helium by a factor of $4 \times 1.6^{2}=10$. Hence, our observations are consistent with our simplified model under the caveats outlined in Section 3.3.2.

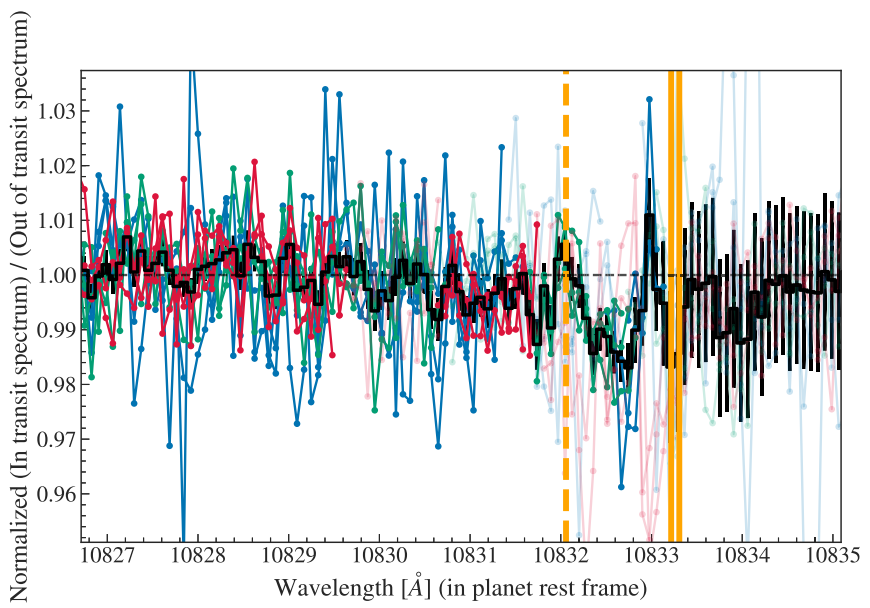

Figure 5. Individual in-transit by out-of-transit ratio HPF spectrum of all nine epochs from the three transits are shown here. Blue curves are the three spectra from the first transit, green are the second transit spectra, and red are the third transit spectra. The black curve is the weighted average with error bars of all the in-transit by out-of-transit spectra. Translucent parts of the curves are telluric and sky emission line corrected regions in each spectrum. They are deweighted to remove any systematic artifacts from influencing the final average. The $x$-axis shows vacuum wavelength in the planet's rest frame at midtransit. The rest of the vacuum wavelengths of the He $10830 \AA$ triplet lines in the planet's rest frame are marked by the vertical dashed and solid orange lines.

\subsection{On the Difference in Model Line Profile and the Observed Absorption Signature}

We discuss a few possible reasons for differences in the shape of the simple model and observed line profile below.

Stellar Activity: Some M dwarfs can be active, and exhibit time variability in the He 10830 absorption line (we note that not all $\mathrm{M}$ dwarfs are active). In comparison to other transmission spectroscopy lines like Ca II K, $\mathrm{Na} \mathrm{I} \mathrm{D,} \mathrm{and} \mathrm{H} \alpha$, active regions typically have He 10830 in absorption (Cauley et al. 2018). Thus, when a planet transits an active spot region on the host star, He 10830 is more likely to result in an emission signature, than an absorption signature. Thus $\mathrm{He}$ 10830 absorption signatures in-transit spectroscopy are more likely to originate in the exosphere than from the stellar chromosphere. However, under high density chromosphere conditions (Andretta \& Giampapa 1995), or active flares, it is possible to obtain He 10830 in emission from the recombination cascade of ionized helium (Huenemoerder \& Ramsey 1987). We do not see any indication of flares during transit in the Ca II IR triplet lines. If the absorption we detected was due to GJ 3470b transiting a very active emission spot, we would expect the absorption signal to not be present in all the in-transit spectra taken (see Figure 5). We have a total of nine in-transit spectra, (three spectra from three transits each). Since the phase of each spectrum is slightly different, it is improbable that there was an unusually strong emission spot of He 10830 during each of our transits. Hence, stellar activity cannot explain the absorption signal we detected during transit of GJ3470b. Stellar activity, however, could probably explain some of the emission structures, and there by the net absorption strength variations we measured across the three transits.

Telluric Absorption and Sky Emission: Systematics in telluric and sky emission line modeling could potentially cause profile variations in the regions with high error bars in Figure 1. The regions with small error bars are free of any telluric or sky emission line contamination in at least one epoch out of the 
three transit observations. Hence, imperfect telluric line modeling errors cannot explain the absorption signature.

Stellar Wind: Even though our simple model, which includes the orbital dynamics and the hydrodynamic outflow velocity, could explain the large blueshifted profile seen in the data, it does not fully explain the shape of the detected line. Some component of this discrepancy could be variability; however, we suspect the stellar wind to play a major role. Since stellar wind accelerates the exosphere toward Earth like a comet tail, it could further blueshift the exosphere at the base of the exosphere skewing the line profile to shorter wavelengths. Further monitoring of the GJ 3470b's He 10830 signature during transits will reveal greater insights on the system.

\section{Conclusion}

We report evidence for He $10830 \AA$ absorption from metastable helium atoms in the base of the outflowing exosphere of the M-dwarf planet GJ 3470b in three transits using the HPF near-infrared spectrograph at the $10 \mathrm{~m} \mathrm{HET} \mathrm{at} \mathrm{the} \mathrm{McDonald}$ Observatory. This measurement marks the first evidence for $\mathrm{He}$ 10830 absorption in an M-dwarf planet. Further, we detect the helium absorption in the velocity range of -36 to $+9 \mathrm{~km} \mathrm{~s}^{-1}$, marking the largest blueshift of He 10830 absorption reported so far in the literature. From our observed absorption, we measure an $\mathrm{EW}$ of $\mathrm{EW}=0.012 \pm 0.002 \AA$, corresponding to a disk surface flux-averaged column density of $N_{\mathrm{He}}^{2} \mathrm{~s}=2.4 \times 10^{10} \mathrm{~cm}^{-2}$. Both the velocity range and the column density we measure are consistent with our exosphere model based on the work of Salz et al. (2016) and Oklopčić \& Hirata (2018) to within the model uncertainties of the UV and X-ray flux of GJ 3470. These observations suggest that detection of the He 10830 absorption around hot Neptunes is now within reach of ground-based facilities-opening up a new window to probe these systems. Puzzling aspects of the broad absorption seen remain, and we have attempted to discuss their source. We recommend future ground- and space-based observations to probe the physics of this absorption signal at even higher signal to noise.

The authors wish to thank M. Salz and A. Oklopčić for discussions that contributed to this work. We thank the anonymous referee for a thoughtful reading and suggestions that improved the quality of the manuscript.

This work was partially supported by funding from the Center for Exoplanets and Habitable Worlds. The Center for Exoplanets and Habitable Worlds is supported by the Pennsylvania State University, the Eberly College of Science, and the Pennsylvania Space Grant Consortium. We acknowledge support from NSF grants AST-1006676, AST-1126413, AST-1310885, AST-1517592, AST-1310875, the NASA Astrobiology Institute (NAI; NNA09DA76A), PSARC, and NIST in our pursuit of precision radial velocities in the NIR. Computations for this research were performed on the Pennsylvania State University's Institute for CyberScience Advanced CyberInfrastructure (ICS-ACI). We acknowledge support from the Heising-Simons Foundation via grant 20170494. This work was supported by NASA Headquarters under the NASA Earth and Space Science Fellowship Program through grants NNX16AO28H and 80NSSC18K1114. Part of this research was carried out at the Jet Propulsion Laboratory, California Institute of Technology, under a contract with NASA.
These results are based on observations obtained with the Habitable-zone Planet Finder Spectrograph on the HobbyEberly Telescope. We thank the Resident astronomers and Telescope Operators at the HET for the skillful execution of our observations of our observations with HPF. The HobbyEberly Telescope is a joint project of the University of Texas at Austin, the Pennsylvania State University, Ludwig-Maximilians-Universität München, and Georg-August Universität Gottingen. The HET is named in honor of its principal benefactors, William P. Hobby and Robert E. Eberly. The HET collaboration acknowledges the support and resources from the Texas Advanced Computing Center.

This research has made use of NASA's Astrophysics Data System Bibliographic Services.

Facility: HET (HPF).

Software: barycorrpy (Kanodia \& Wright 2018), astropy (Astropy Collaboration et al. 2018), numpy (van der Walt et al. 2011), scipy (Jones et al. 2001), matplotlib (Hunter 2007), CoCalc (SageMath 2019), GNU parallel (Tange 2011).

\section{ORCID iDs}

Joe P. Ninan (1D https://orcid.org/0000-0001-8720-5612 Gudmundur Stefansson (iD https://orcid.org/0000-00017409-5688

Suvrath Mahadevan (10) https://orcid.org/0000-00019596-7983

Chad Bender (iD https://orcid.org/0000-0003-4384-7220

Paul Robertson (1D https://orcid.org/0000-0003-0149-9678

Ryan Terrien (i) https://orcid.org/0000-0002-4788-8858

Jason Wright (ib https://orcid.org/0000-0001-6160-5888

Shubham Kanodia (ib https://orcid.org/0000-0001-8401-4300

William Cochran (iD https://orcid.org/0000-0001-9662-3496

Michael Endl (1) https://orcid.org/0000-0002-7714-6310

Eric B. Ford (ib https://orcid.org/0000-0001-6545-639X

Samuel Halverson (iD https://orcid.org/0000-0003-1312-9391

Arpita Roy (ib https://orcid.org/0000-0001-8127-5775

Christian Schwab (i) https://orcid.org/0000-0002-0091-7105

\section{References}

Allart, R., Bourrier, V., Lovis, C., et al. 2018, Sci, 362, 1384

Allart, R., Bourrier, V., Lovis, C., et al. 2019, A\&A, 623, A58

Alonso-Floriano, F. J., Snellen, I. A. G., Czesla, S., et al. 2019, A\&A, 629, A110

Andretta, V., \& Giampapa, M. S. 1995, ApJ, 439, 405

Astropy Collaboration, Price-Whelan, A. M., Sipőcz, B. M., et al. 2018, AJ, 156,123

Awiphan, S., Kerins, E., Pichadee, S., et al. 2016, MNRAS, 463, 2574

Bender, C. F., Mahadevan, S., Deshpande, R., et al. 2012, ApJL, 751, L31

Biddle, L. I., Pearson, K. A., Crossfield, I. J. M., et al. 2014, MNRAS, 443, 1810

Bourrier, V., Lecavelier des Etangs, A., Ehrenreich, D., et al. 2018, A\&A, 620, A147

Bray, I., Burgess, A., Fursa, D. V., \& Tully, J. A. 2000, A\&AS, 146, 481

Brown, R. L. 1971, ApJ, 164, 387

Cauley, P. W., Kuckein, C., Redfield, S., et al. 2018, AJ, 156, 189

Clough, S., Shephard, M., Mlawer, E., et al. 2005, JQSRT, 91, 233

Dere, K. P., Landi, E., Mason, H. E., Monsignori Fossi, B. C., \& Young, P. R. 1997, A\&AS, 125, 149

Dere, K. P., Landi, E., Young, P. R., et al. 2009, A\&A, 498, 915

Dragomir, D., Benneke, B., Pearson, K. A., et al. 2015, ApJ, 814, 102

Drake, G. 2006, in High Precision Calculations for Helium, ed. G. Drake (New York: Springer), 199

Drake, G. W. F. 1971, PhRvA, 3, 908

Eggleton, P. P. 1983, ApJ, 268, 368

Ehrenreich, D., Bourrier, V., Wheatley, P. J., et al. 2015, Natur, 522, 459

Gaia Collaboration, Brown, A. G. A., Vallenari, A., et al. 2018, A\&A, 616, A1 
Hairer, E., \& Wanner, G. 1996, Solving Ordinary Differential Equations. II. Stiff and Differential-Algebraic Problems (Berlin: Springer)

Huenemoerder, D. P., \& Ramsey, L. W. 1987, ApJ, 319, 392

Hunter, J. D. 2007, CSE, 9, 90

Jones, E., Oliphant, T., Peterson, P., et al. 2001, SciPy: Open Source Scientific Tools for Python, 22, http://www.scipy.org/

Kanodia, S., \& Wright, J. 2018, RNAAS, 2, 4

Kaplan, K. F., Bender, C. F., Terrien, R., et al. 2018, in ASP Conf. Ser. 523, Astronomical Data Analysis Software \& Systems XXVII, ed. P.J. Teuben (San Francisco, CA: ASP), 567

Kosiarek, M. R., Crossfield, I. J. M., Hardegree-Ullman, K. K., et al. 2019, AJ, 157, 97

Kulow, J. R., France, K., Linsky, J., \& Loyd, R. O. P. 2014, ApJ, 786, 132

Linsky, J. L., Fontenla, J., \& France, K. 2014, ApJ, 780, 61

Linsky, J. L., France, K., \& Ayres, T. 2013, ApJ, 766, 69

Mahadevan, S., Ramsey, L. W., Terrien, R., et al. 2014, Proc. SPIE, 9147, $91471 \mathrm{G}$

Mansfield, M., Bean, J. L., Oklopčić, A., et al. 2018, ApJL, 868, L34

Metcalf, A. J., Anderson, T., Bender, C. F., et al. 2019, Optica, 6, 233

Moutou, C., Coustenis, A., Schneider, J., Queloz, D., \& Mayor, M. 2003, A\&A, 405, 341

Ninan, J. P., Bender, C. F., Mahadevan, S., et al. 2018, Proc. SPIE, 10709, $107092 \mathrm{U}$

Norcross, D. W. 1971, JPhB, 4, 652

Nortmann, L., Pallé, E., Salz, M., et al. 2018, Sci, 362, 1388
Oklopčić, A., \& Hirata, C. M. 2018, ApJL, 855, L11

Osterbrock, D. E., \& Ferland, G. J. 2006, Astrophysics Of Gas Nebulae and Active Galactic Nuclei (Mill Valley, CA: Univ. Science Books)

Pizzolato, N., Maggio, A., Micela, G., Sciortino, S., \& Ventura, P. 2003, A\&A, 397, 147

Ramsey, L. W., Adams, M. T., Barnes, T. G., et al. 1998, Proc. SPIE, 3352, 34 Roberge, W., \& Dalgarno, A. 1982, ApJ, 255, 489

SageMath, I. 2019, CoCalc Collaborative Computation Online, https:// cocalc.com/

Salz, M., Czesla, S., Schneider, P. C., et al. 2018, A\&A, 620, A97

Salz, M., Czesla, S., Schneider, P. C., \& Schmitt, J. H. M. M. 2016, A\&A, 586, A75

Seager, S., \& Sasselov, D. D. 2000, ApJ, 537, 916

Shetrone, M., Cornell, M. E., Fowler, J. R., et al. 2007, PASP, 119, 556

Spake, J. J., Sing, D. K., Evans, T. M., et al. 2018, Natur, 557, 68

Stefansson, G., Cañas, C., Wisniewski, J., et al. 2020, AJ, 159, 100

Stefansson, G., Hearty, F., Robertson, P., et al. 2016, ApJ, 833, 175

Stone, J. M., \& Proga, D. 2009, ApJ, 694, 205

Tange, O. 2011, login: The USENIX Magazine, 36, 42, http://www.gnu.org/ $\mathrm{s} /$ parallel

Turner, J. D., Christie, D., Arras, P., Johnson, R. E., \& Schmidt, C. 2016, MNRAS, 458, 3880

van der Walt, S., Colbert, S. C., \& Varoquaux, G. 2011, CSE, 13, 22

Vidal-Madjar, A., des Etangs, A. L., Désert, J.-M., et al. 2003, Natur, 422, 143 